\title{
Microvascular Task Trainer in Neurosurgical Residency Training: A Pilot Study
}

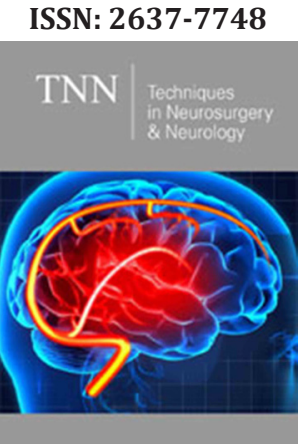

*Corresponding author: Elsa Olson, University of Illinois, USA

Submission: 悳 December 19, 2020

Published: 笽 February 01, 2021

Volume 4 - Issue 1

How to cite this article: CPT Callum DD, Elsa Olson,Jake Stewart, Carter Bell, Jonathan Garst andJulian Lin. Microvascular Task Trainer in Neurosurgical Residency Training: A Pilot Study. Tech Neurosurg Neurol. 4(1). TNN. 000577. 2021.

DOI: $10.31031 / \mathrm{TNN} .2021 .04 .000577$

Copyright@ Elsa Olson, This article is distributed under the terms of the Creative Commons Attribution 4.0 International License, which permits unrestricted use and redistribution provided that the original author and source are credited.

\author{
CPT Callum DD ${ }^{1}$, Elsa Olson ${ }^{1 *}$, Jake Stewart ${ }^{1}$, Carter Bell ${ }^{2}$, Jonathan Garst ${ }^{1,3}$ and \\ Julian Lin ${ }^{3,4}$ \\ ${ }^{1}$ University of Illinois College of Medicine, USA \\ ${ }^{2}$ Harvard College, Boston, USA \\ ${ }^{3}$ OSF Saint Francis Department of Neurosurgery, USA \\ ${ }^{4}$ Illinois Neurological Institute, USA
}

\begin{abstract}
Background: The STA-MCA bypass is a technically challenging procedure that many resident neurosurgeons fail to garner experience with during training. Our 3D-printed micro-vessel task trainer is designed to create a user friendly, portable, multi-vessel compatible suturing training protocol for neurosurgical residents.
\end{abstract}

Objective: Goals are 1) determine the capacity of the device to discriminate skill level of residents and 2) use objective measures to analyze improvements in performance across trials to provide evidence for task trainer efficacy.

Method: Neurosurgical resident suturing trials using bio-synthetic vessels and trainers are scheduled, performed and recorded in OR with Zeiss microscope. Anastomosis leakage is measured, and videos are analyzed with blinded Northwestern Modified Objective Assessment Tool (NOMAT).

Result: In 2019, approximately 20 hours of $2 \mathrm{~mm}$ vessel anastomoses sessions with 9-0 and $10-0$ sutures used among 7 neurosurgical residents with various experiences are analyzed and recorded. Each resident averaged 4.7 anastomoses sessions: range 2-7. We used a paired t-tests to compare individual performance changes, and Kruskal-Wallis test to compare PGY group (PGY1-2, 3-4, 5-6) performance. The two-tailed p-values were calculated using SAS. Final NOMAT scores were statistically higher than baseline by 19.7 points $(p=0.035, s d=19.2)$. An inverse relationship exists between NOMAT improvement and PGY level, as well as absolute time reduction from final trial to baseline. A negative correlation exists between number of trials and time to completion (Spearman correlation coefficient=-0.93, $\mathrm{p}=0.0026$ ).

Conclusion:Unique task trainer-based simulation in learning micro-anastomoses suturing is advantageous and can be incorporated into resident's curriculum. Video analysis of trials documents performance, progression and improvement of performance.

\section{Introduction}

Motor learning progresses through controlled repetition. For centuries, simulation has been utilized in the education and training of professionals in the fields of medicine, aerospace, nuclear power and aviation [1]. For each of these fields, real world training is either too dangerous or too costly. The training for small vessel neurosurgery is no exception. End-to-end anastomoses is a revascularization technique useful in treatment of cerebral hemodynamic failure and complex aneurysm surgery. Traditionally, vascular training for these tasks was accomplished using animal models, with inherent challenges of medical ethics, anesthesia, and the costs of pre-and post-surgical care. The current method of surgical training for most residency programs is based on the apprenticeship model - first introduced by Dr. William Halsted in the early 20th century. It is based on learning by direct observation and imitation of the mentor's actions. The field of medicine now, however, has advanced tools for learning that have the potential to overcome impediments to education [2] including litigation, resident work hour restrictions, and high operating room stress for surgical trainees, which limit the effectiveness of the Halsted model. Simulation task trainers offer alternative methods for developing motor dexterity and cognitive skills necessary to successfully perform procedures that are not readily available to learners such as the microvascular anastomosis $[3,4]$. Limited clinical time also restricts learners' preparedness and amplifies stress pertaining to perceptions of efficient skill levels. 


\section{Methods}

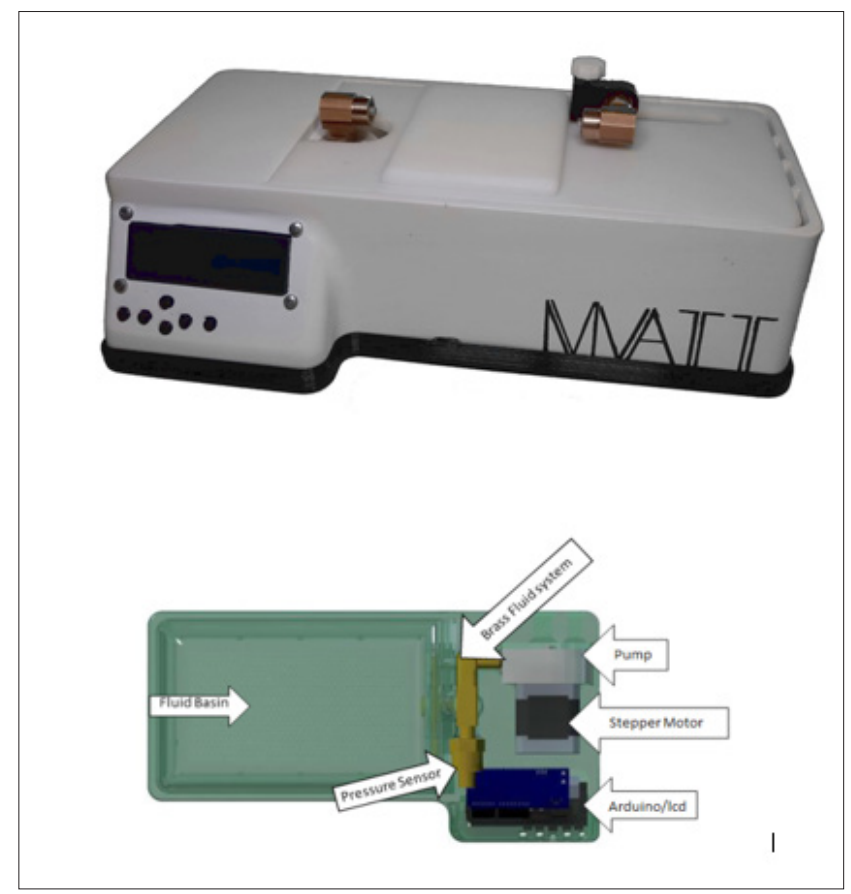

Figure 1: Microvascular task trainer with user interface and suturing platform. The 3D model rendering illustrates the individual components of the device, including fluid basin, pump, stepper motor, pressure sensor and LCD control.

In collaboration with Bradley University Mechanical Engineering Department and as a part of the Senior Design Project, our designed small vessel task trainer, (Figure 1) has the capacity to be equipped with Life Like's (Life Like Biotissue Ontario, Canada) $1 \mathrm{~mm}, 2 \mathrm{~mm}$, or $3 \mathrm{~mm}$ microvessel. The microvessel measurements pertain to the outside diameter ( $1 \mathrm{~mm}, 2 \mathrm{~mm}$ and $3 \mathrm{~mm}$ ), with a lumen size of $0.8,1.5$ and $2.3 \mathrm{~mm}$ respectively. For the purposes of this study, participants solely utilized the $2 \mathrm{~mm}$ microvessels. Each volunteer resident participant performed a series of small vessel suturing procedures that simulates the microvascular anastomoses. Individual performances were graded using a modified NOMAT, Table 1 . Times to completion were recorded for all trials. Each trial was timed from the first instrument touch to the point at which the vessel was repaired and flow established [5]. Anonymous hand video-monitoring via the Zeiss microscope, recorded all performed procedures. Initial fidelity of the small vessel suturing trainer was assessed by an attending neurosurgeon. Each trial was performed within the surgical operating room, utilizing operating room protocols and strict aseptic technique. The first trial for each procedure constituted the subject's performance baseline. The ensuing trials consisted of the practice period, during which each resident participant had unlimited access to the task trainer and vessels. For each timed practice trial, we additionally measured leak and NOMAT score. At the time when the participants deemed themselves prepared (self-judgement), they performed a final video-recorded trial. The video-recordings were then viewed, analyzed and graded for each simulation task using the aforementioned modified NOMAT by an expert observer. The video recordings were limited to a view of only the resident's gloved hands, eliminating potential identifying bias [6-11]. The modified NOMAT grading scale contained ten grading fields, scored on a Likert scale of one to ten $[4,12]$. Time to completion represented the time from first instrument touch to reestablishment of flow to a patent vessel. The study was approved by our IRB. We used paired t-test to compare the overall outcome changes, and Kruskal-Wallis test to compare the changes among the three PGY groups. The twotailed $p$ values were calculated for all tests using SAS and $p<0.05$ is considered the statistically significant test. All statistical analyses were performed using SAS 9.4(SAS Institute Inc, Cary, NC).

Table 1: Modified northwestern objective microanastomoses assessment tool (NOMAT), evaluated on a 10pt Likert scale.

\begin{tabular}{|c|}
\hline Use of Microscope \\
\hline Handling of surgical instruments \\
\hline Vessels handling and respect for tissue \\
\hline Needle handling and care \\
\hline Needle bite uniformity \\
\hline Spacing of the sutures \\
\hline Knot tying \\
\hline Needle efficiency \\
\hline Knot tying efficiency \\
\hline Off pump evaluation of complete anastomoses \\
\hline
\end{tabular}

\section{Result}

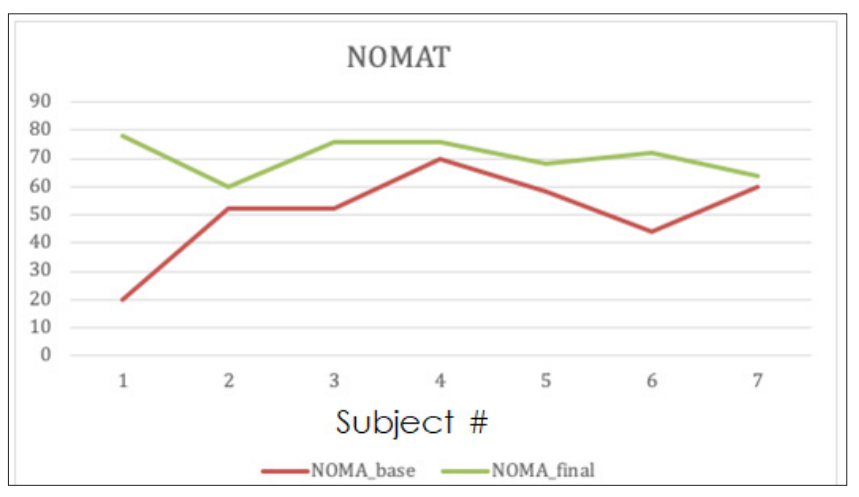

Figure 2: Line graphs of baseline vs final NOMAT scores for each individual resident participant.

Seven of ten neurosurgical residents from the University of Illinois College of Medicine Peoria volunteered to participate in the study. Three residents were placed in the novice group (PGY12; 42.9\%), 2 residents were placed in the intermediate (PGY3-4; 28.6\%) and expert (PGY5-6; 28.6\%) groups, respectively. The average number of trials of the novice group was 6 , intermediate 5.5 and expert was 3. No statistical difference is present in the number of trials. Line graphs of baseline vs final NOMAT scores for 
each individual resident participant is shown in Figure 2. Sample size estimation and power analysis are not needed for this pilot study. After checking the normality assumption, we used paired t-test to compare differences between baseline and post values of time to completion, and modified NOMAT overall scores. Modified NOMAT scores analysis on all subjects $(\mathrm{N}=7)$ showed that final NOMAT scores were higher than baseline scores $(\mathrm{P}=0.035)$, with average NOMAT improvement of 19.7 points with a standard deviation of 19.2. Comparison of improvement across three PGY groups showed the novice group improved the most, but with no statistically significant difference $(\mathrm{P}=0.3679)$. Paired $\mathrm{T}$ tests showed that Final trial times were faster than baseline scores $(\mathrm{P}=0.047)$. Average time improvement across all subjects was 933.4 secs with sd of 990.1 secs. Comparison of time improvement across three groups showed trending results of greater improvement in the novice group compared to other groups $(\mathrm{P}=0.1403)$. Time improvement distributions are displayed in Figure 3. Analysis comparing the association between number of trials and NOMAT improvement using spearman correlation coefficients showed a positive correlation the higher the number of trials, the larger the improvement in NOMAT (spearman correlation coefficient 0.27 , $\mathrm{P}=0.554$ ). The correlation coefficient between time improvement and number of trials displayed a negative correlation-the more practice trials, the greater the time improvement $(-0.93, \mathrm{P}=0.0026)$.

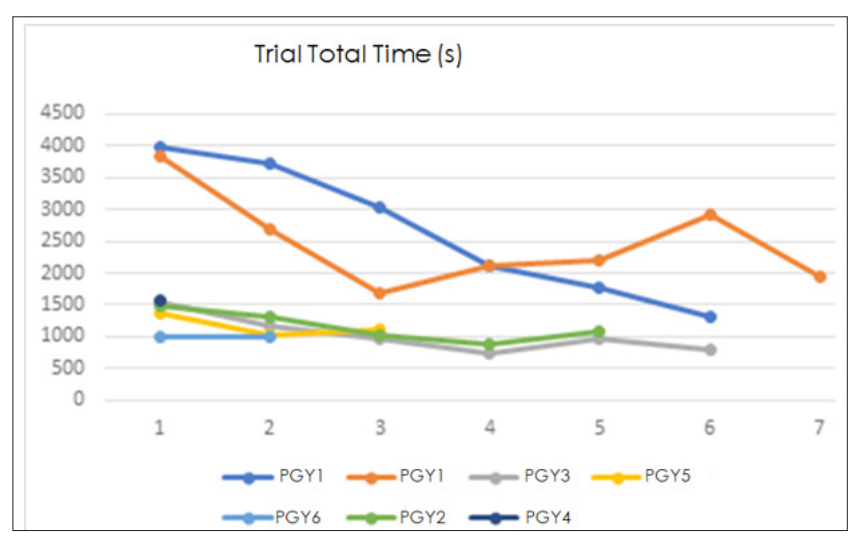

Figure 3: Line graphs of each resident and individual time records in seconds per trial.

\section{Discussion}

The purpose of this study was to determine the efficacy of a multi-vessel compatible task trainer built and designed by team members. The study was created as an alternate to the 2015 model devised at Northwestern University,7 with the Northwestern Objective Microanastomosis Assessment Tool (NOMAT). Our study modified the NOMAT criteria and utilized our unique task trainer to analyze baseline ability of each tested neurosurgical resident, and also analyzed improvement through motor repetition. The unique quality and design of our specialized trainer, Figure 1 allowed the user full control of flow rate and pressure within the fixed circuit. User interface adjusted flow from $5 \mathrm{~mL} / \mathrm{min}$ to $1 \mathrm{~L} /$ min and pressure from 50 to $170 \mathrm{mmHg}$. Built-in pressure sensors allowed for flow termination upon upstream clamping of vessels. Custom designed operating surface and vessel attachment points accommodated vessel sizes ranging from $1 \mathrm{~mm}$ to $3 \mathrm{~mm}$ in diameter. Each component of the device was detachable and replaceable; all developed using 3D polyamide printing materials. The portability of this task trainer gave the user the freedom to hone skills in a preferred environment 1,5 The trainer can additionally be used as a surgical warm-up tool immediately prior to the patient's surgery.

The primary endpoints of both time and NOMAT improvement across all residents $(\mathrm{N}=7)$ showed statistically significant improvement from baseline to final trials. However, despite trending results, there was no significant difference across groups in either endpoint. The lack of significance is likely due to our small sample size. We would expect the novice groups to have larger improvement potential compared to the more experienced residents. As one would expect, an increased number of practice trials was associated with both time improvement and NOMAT improvement. Limitations of the study include a low $\mathrm{N}$ due to the size of the medical campus's neurosurgical residency program. Resident feedback revealed areas for improvement in the device hardware. Future device iterations will focus on improving the ergonomics of the trainer with extensions for stability hand rests, as well as incorporating additional distal outlet flow for multiple end-to-side anastomosis and a dural suturing platform. Beyond hardware adjustments, to increase the $\mathrm{N}$ of this study, we have the capacity of expanding to the other medical campuses, as well as recruiting interdepartmentally.

\section{Conclusion}

Utilizing the small vessel task trainer as a complementary teaching tool provides the low risk and low stress learning environment currently lacking in the neurosurgical suturing training protocol. The portability of the trainer creates capacity for a novel, pre-surgical warmup in the operating room. Video analysis of training sessions documents performance, progression, and improvement across trials. This unique trainer allows for early use and experience with the Ziess microscope as early as PGY1, allowing for expedited practice with handling 1-3mm microvessels starting from day 1 of residency. The vessel versatility and portability of the simulator provides the potential to be incorporated into all surgical fields. This study displays the potential for improvement in microanastomosis through motor learning through repetition, with the greatest improvements attainable early in neurosurgical residency.

\section{Acknowledgement}

We would like to thank Dr. Hannah Wang for her statistical expertise, and the engineering team at Bradley University for their assistance with creation of our trainer.

\section{References}

1. Bradley Paul (2006) The history of simulation in medical education and possible future directions. Med Educ 40(3): 254-262. 
2. Kneebone, Roger (2003) Simulation in surgical training: educational issues and practical implications. Med Educ 37(3): 267-277.

3. Nesbitt, Craig Iain (2016) The role of simulation in the development of endovascular surgical skills. Perspect Med Educ 5(1): 8-14.

4. Price Joel (2011) A randomized evaluation of simulation training on performance of vascular anastomosis on a high-fidelity in vivo model: the role of deliberate practice. J Thorac Cardiovasc Surg 142(3): 496503.

5. Fann James (2008) Improvement in Coronary anastomosis with cardiac surgery simulation. J Thorac Cardiovasc Surg 136(6): 1486-1491.

6. Black (2007) Training for carotid intervention: preparing the next generation. Eur J Vasc Endovasc Surg 33(5): 518-524.

7. Hong, Jong Won (2010) Evaluation of the efficacy of microsurgical practice through time factor added protocol. J Craniofac Surg 21(3): 876-881.

8. Mcgaghie, William C (2016) A critical review of simulation-based medical education research: 2003-2009. Med Educ 50(10): 986-991.

9. Birkmeyer, John D (2013) Surgical skill and complication rates after bariatric surgery. N Eng J Med 369(15): 1434-1442.

10. Ghasemloonia, Ahmad (2017) Surgical skill assessment using motion quality and smoothness. J Surg Educ 74(2): 295-305.

11. Uemura, Munenori (2014) Analysis of hand motion differentiates expert and novice surgeons. J Surg Res 188(1): 8-13.

12. Wade T, Webb (2012) Tackling technical skills competency: a surgical skills rating tool. J Surg Res 172(2): 293.

For possible submissions Click below: 